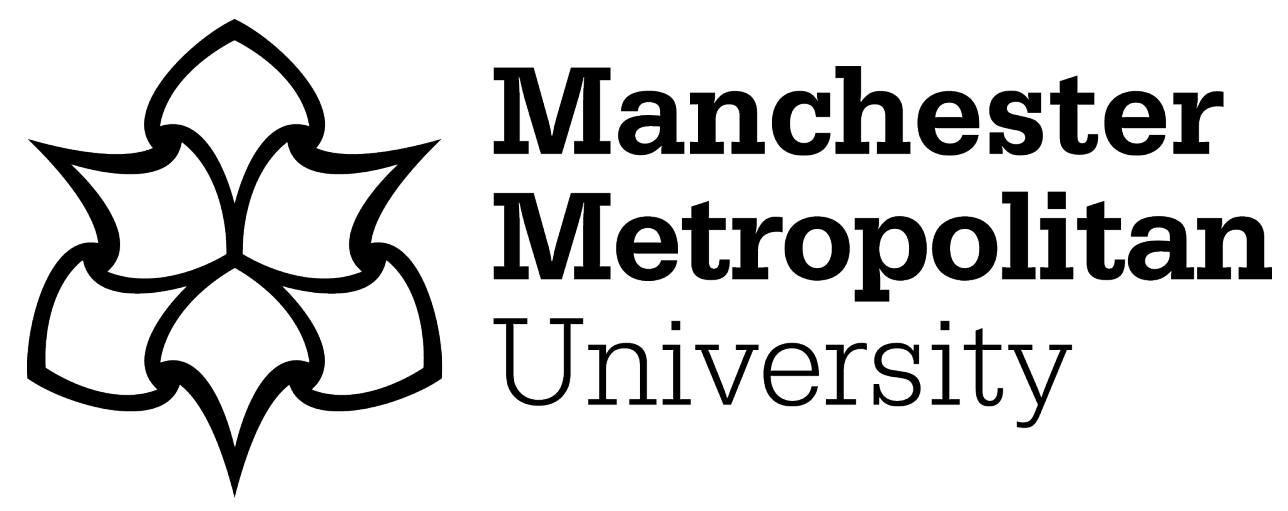

Twati, WA, Wood, DJ, Liskiewicz, TW, Willmott, NS and Duggal, MS (2009) An evaluation of the effect of non-setting calcium hydroxide on human dentine: a pilot study. European archives of paediatric dentistry : official journal of the European Academy of Paediatric Dentistry, 10 (2). pp. 104-109. ISSN $1818-6300$

Downloaded from: https://e-space.mmu.ac.uk/625930/

Version: Accepted Version

Publisher: Springer

DOI: https://doi.org/10.1007/BF03321610

Please cite the published version 


\title{
An Evaluation of the Effect of Non-Setting Calcium Hydroxide on Human Dentine: A pilot Study
}

\author{
W. A. Twati*, D. J. Wood*, T. W. Liskiewicz**, N. S. Willmott*, M. S. Duggal * \\ ${ }^{*}$ Dept. Paediatric Dentistry, Leeds Dental Institute, **School of Mechanical Engineering. University of Leeds, Leeds, England.
}

\begin{abstract}
AIM: To evaluate the effect of non-setting calcium hydroxide $(\mathrm{NSCH})$ on the hardness and elastic modulus of dentine from extracted permanent premolar human teeth. METHODS: 30 freshly extracted single rooted human premolar teeth were decoronated and the roots then sectioned longitudinally into equal halves. In the experimental group a thin layer of $\mathrm{NSCH}$ was applied whilst the control group had no medicament. After 1, 3 and 6 months, nanoindentation was used to assess dentine hardness and the modulus of elasticity. Scanning Electron Microscopy (SEM) was used to visualize the depth of penetration of $\mathrm{NSCH}$ into the dentinal tubules. RESULTS: SEM images showed that there were no structural changes in the dentine slabs that had NSCH application after 1, 3 or even 6 months. However, penetration of $\mathrm{NSCH}$ into the dentine tubules was seen at both 3 and 6 months with a significant reduction in the hardness of dentine observed at $3(p<0.02)$ and 6 months $(p<0.01)$. The modulus of elasticity was significantly lower $(p<0.01)$ at 6 months. CONCLUSION: It appears that there is a significant reduction in the hardness of dentine with increasing periods of calcium hydroxide application. Prolonged application of $\mathrm{NSCH}$ could have a detrimental effect on dentine, making the dentine more prone to fracture.
\end{abstract}

\section{Introduction}

At present, the management of non-vital immature incisors requires complete elimination of bacteria, necrotic pulp, tissue debris and the use of a suitable material to induce an apical hard tissue barrier in order to facilitate obturation. Calcium hydroxide $\left(\mathrm{CaOH}_{2}\right)$ was introduced as early as 1920 , [Hermann, 1920] and it was suggested very early on that this material mixed with camphorated parachlorophenol (CMCP) would induce a calcified barrier across the apex [Kaiser, 1964]. This material became popular when it was observed that $\mathrm{CaOH}_{2}$ also reduced bacterial contamination of the root canals [Frank, 1966].

According to Kinirons et al. [2001] the mean time taken for non-setting calcium hydroxide (NSCH) to induce an apical hard tissue barrier is 9 months. However, it has been pointed out that long-term use of $\mathrm{NSCH}$ in non-vital immature incisors might lead to a weakening of the dentine structure due to degradation of the proteins [Andreasen et al., 2002]. This may make the dentine more susceptible to fracture. High incidences of root fracture have been reported for non-vital immature permanent incisors that have been managed endodontically, particularly with NSCH [Cvek, 1992; Al-Jundi, 2004]. In a more recent study, Rosenberg et al. [2007] showed a significant reduction in the mean tensile fracture strength (MTFS) of dentine with prolonged use of $\mathrm{NSCH}$. Given these preliminary reports it seemed prudent for dental researchers to study the effect of $\mathrm{NSCH}$ on the physical properties of human dentine.

The aim of this in vitro pilot study, therefore, was to evaluate the effect of $\mathrm{NSCH}$ on the hardness and modulus of elasticity of human dentine from extracted human permanent teeth.

\section{Materials and methods}

Samples. Following patient informed consent, 30 extracted permanent single rooted human premolar teeth were collected. The teeth had been extracted from adolescents and young adults, aged $10-18$ years of age for orthodontic reason. Each tooth was cleaned with distilled water and all remnants of soft tissue were removed with a scaler. The teeth were then stored in water with a crystal of thymol added. The teeth were decoronated and sectioned longitudinally into equal halves with a Precision Diamond Wire Saw. (Well, USA). (Figure1a).

One of the pair of samples was used as a control, with no medicament, and the other was covered with a layer of $\mathrm{NSCH}$ (UltraCal XS, Ultradent, USA). The samples were then placed in a petri-dish alongside saline soaked gauze and incubated at $37^{\circ} \mathrm{C}$. The wet gauge ensured a humid environment around the samples whilst they were in the incubator. The gauze was changed weekly. At 1,3 and 6 months 10 paired-samples (10 experimental samples and the 10 corresponding control samples) were investigated as follows:

- Preparation of samples. 5 experimental samples plus 5 corresponding control samples were polished, etched and sputter coated with gold,

- SEM was used for examination,

- Nanoindentation. 5 experimental samples plus 5 corresponding control samples were embedded in composite resin using a custom made mould, which, because the samples were hemi-sectioned, was necessary to create a 
flat surface to facilitate measurement. A small hole placed in the bottom of the die enabled the composite block holding the dentine slab to be removed without any damage to the surface that was to be measured (Figure 1b).

The block with an embedded sample was then cut in half transversely across the root as shown in Figure 1c. The "top" exposed surface was thus not used for measurement of hardness rather an internal surface was preferred. Hardness was assessed by Nanoindentation (Micro Material Ltd, Wrexham, UK) using 5 indentations that were spaced evenly over the surface that was to be assessed. The mean of these 5 indentations was then used as the final measurement.

Calculation of Young's Modulus of Elasticity. The value obtained from nanoindentation was used in a formula described by Oliver and Pharr [1992] to calculate the Young's Modulus of Elasticity of Dentine.

The Young's Modulus was calculated from the following equation:

$\frac{1}{E^{*}}=\frac{1-v_{m}^{2}}{E_{m}}+\frac{1-v_{i}^{2}}{E_{i}}$

$\mathrm{Em}=\left(1-v_{m}\right)\left[\frac{1}{E^{*}}-\frac{1-v_{i}^{2}}{E_{i}}\right]^{-1}$

Where: $\mathrm{E}^{\star}$ is the value obtained from nanoindentation; $\mathrm{Em}$ is the modulus of elasticity; Ei has a constant value $=0.07$; Vi has a constant value $=1141 ; \mathrm{Vm}$ obtained from the literature $=0.03$ (Toparli and Koksal, 2005)

Statistical analysis. The mean differences between the two groups and their significance was tested using paired t tests. SPSS (Version 15) was used to calculate the means and confidence intervals and a $p$ value of $p<0.05$ was accepted as statistically significant.

\section{Results}

Scanning Electron Microscope (SEM). The SEM of the control slab is shown in Figure 2a. At 1, 3 and 6 months there was no obvious distortion or destruction of the root dentine for any of the experimental samples compared with the control. After one month of $\mathrm{NSCH}$ application it was noted that the material was present only in the outer layers of dentinal tubules, and no penetration of $\mathrm{NSCH}$ was observed into the dentinal tubules (Figure 2b). However after 3 (Figure 2c) and 6 months (Figure 2d) of the application of $\mathrm{NSCH}$ it can be seen that the $\mathrm{NSCH}$ particles have penetrated into the dentinal tubules.

Nanoindentation. There was no significant difference for hardness or Modulus of Elasticity between the control and experimental samples after one month (Table 1). At 3 months (Table 2) there was a reduction in both the mean hardness and modulus of elasticity of the dentine compared with the
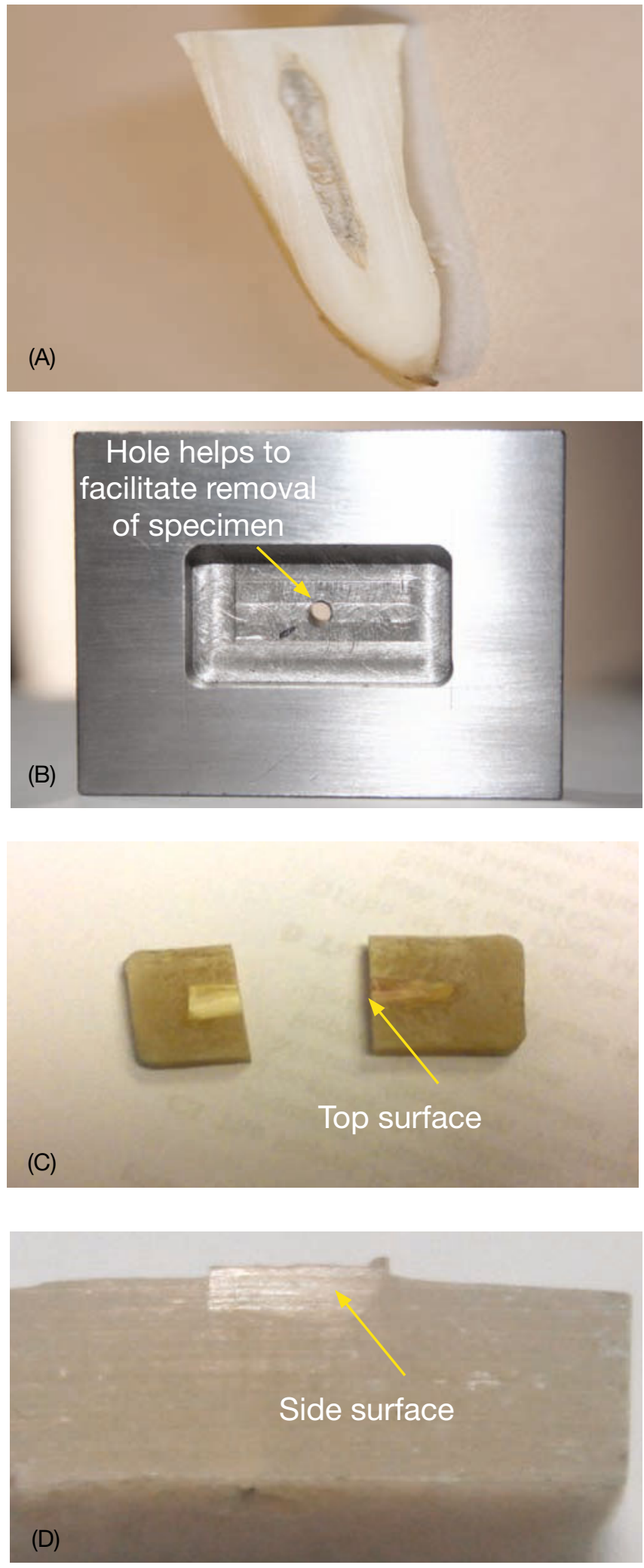

Figure 1. Preparation of samples: A. An example of the sectioned tooth root for both control and experimental groups. B. Metal die used for preparation of specimens. C. Final appearance of experimental sample. D. Prepared specimen showing surface to be measured. 

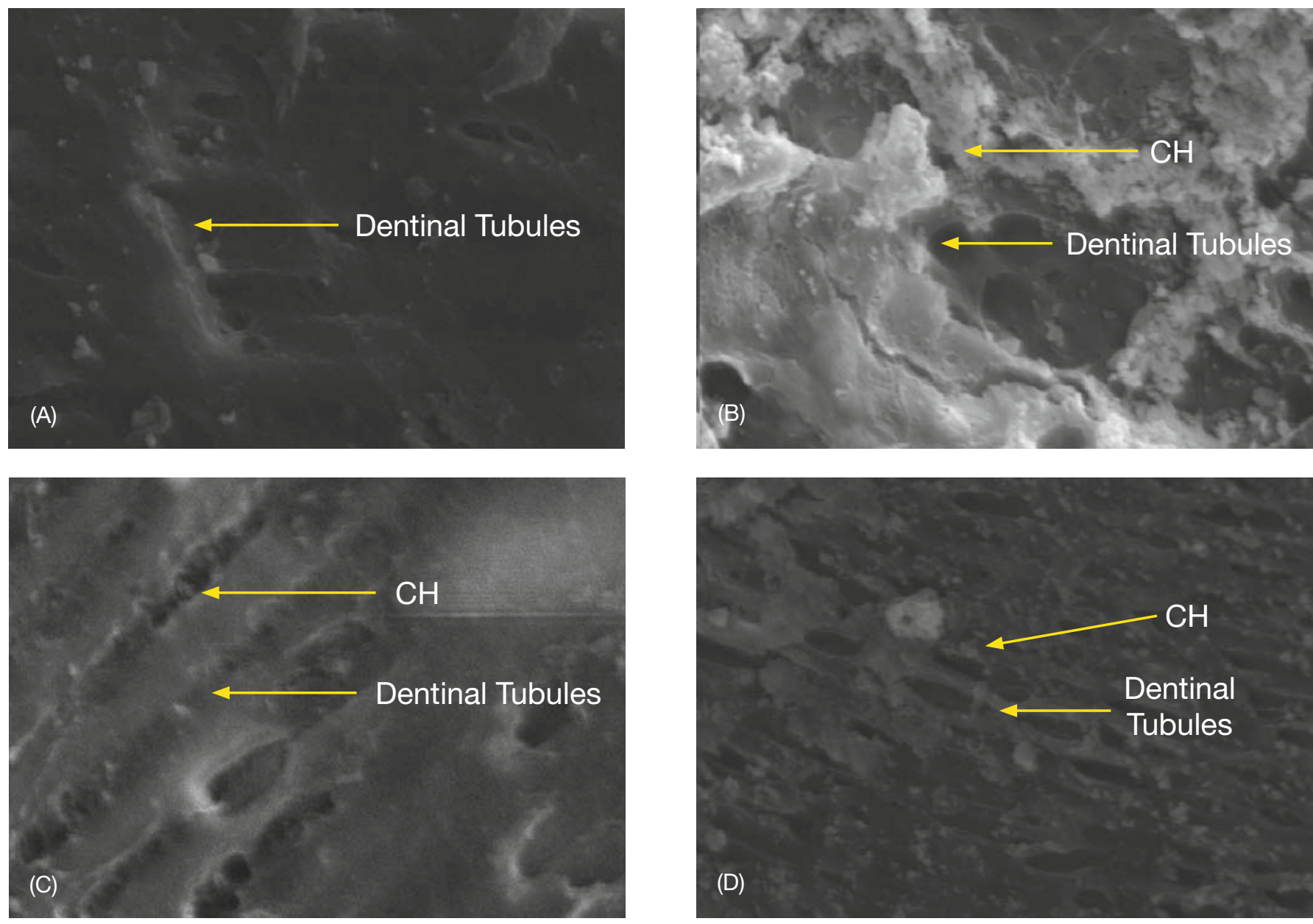

Figure 2. Scanning Electron Microscope micrographs of: A. a control slab with no medicament applied. B. Longitudinal section of an experimental slab one month after application of calcium hydroxide. $\mathbf{C}$. Showing $\mathrm{CaOH}$ particles inside the dentinal tubule three months after application of $\mathrm{CaOH}$. D. Six months after application of $\mathrm{CaOH}$ showing calcium hydroxide penetration in dentinal tubules.

control samples, though this was only statistically significant for hardness $(p<0.02)$. After 6 months (Table 3) there was a further and statistically significant reduction in both the hardness $(p<0.01)$ and modulus of elasticity $(p<0.01)$ compared with the control samples.

\section{Discussion}

Nanoindentation has been used in many recent studies on the mechanical properties of calcified hard tissue [Angker and Swain, 2006]. This method allows a sensitive method to measure the mechanical properties, hardness and elastic modulus for dentine [Chng et al., 2002].

The nanoindentation technique is a non-destructive, simple procedure compared with others and sample preparation is less time consuming. It does however require that the surface to be measured is flat and smooth. The metal die that was used allowed embedding of the dentine slabs for nanoindentation and to develop a flat surface and also provide stability during the process of indentation. The "top" surface (Figure 1a) was ignored for hardness measurement as it was found on the SEM examination of the surfaces that the "top" surface was covered by a smear layer which was about 1015 microns thick, which could have affected the results of nanoindentation. However, it should be pointed out that in this study only measurements of hardness over the surfaces that were studied were assessed. It would have been interesting to also investigate the depth of the effect of $\mathrm{NSCH}$, but that was not the aim of this study.

The surface actually used for measurement was thus only exposed (Fig 1b) after cutting through the composite block. This "internal" surface had a smear layer of less than $5 \mathrm{mi}-$ crons that was considered acceptable for this measurement. Furthermore, the storage media and method could have influenced the dentine properties [Habelitz et al., 2000]. Samples in our study were kept in a humid environment by putting soaked gauze of saline in every Petri dish. The gauze 
Table 1: Hardness values GPa* and Modulus of elasticity of control $\mathrm{GPa}^{\star}$ and corresponding experimental samples at one month for dentine samples treated with non-setting calcium hydroxide.

\begin{tabular}{l|c|c|c|c}
\multirow{2}{*}{ Slabs } & \multicolumn{2}{|c|}{ Control Group $\mathbf{n = 5}$} & \multicolumn{2}{c}{ Experimental group $\mathbf{n = 5}$} \\
\cline { 2 - 5 } & Hardness & Modulus of elasticity & Hardness & Modulus of elasticity \\
\cline { 2 - 5 } & GPa* & GPa & GPa & GPa \\
\hline 1 & 0.77 & 20.70 & 0.77 & 20.70 \\
\hline 3 & 0.78 & 20.24 & 0.65 & 18.98 \\
\hline 4 & 0.66 & 24.62 & 0.71 & 18.98 \\
\hline 5 & 0.80 & 22.11 & 0.77 & 20.70 \\
\hline Mean & 0.79 & 21.19 & 0.68 & 23.36 \\
\hline SD & 0.76 & 21.77 & 0.72 & 20.54 \\
\hline PD & 0.057 & 01.74 & 0.054 & 01.79
\end{tabular}

$\mathrm{P}=$ Not Significant (NS) for Hardness; $\mathrm{P}=$ Not Significant (NS) for Modulus of Elasticity

Table 2: Hardness values $\mathrm{GPa}^{*}$ and Modulus of elasticity $\mathrm{GPa}^{\star}$ of control and corresponding experimental dentine samples at three months after treatment with non-setting calcium hydroxide.

\begin{tabular}{l|c|c|c|c}
\multirow{2}{*}{ Slabs } & \multicolumn{2}{|c|}{ Control Group $\mathbf{n = 5}$} & \multicolumn{2}{c}{ Experimental group $\mathbf{n = 5}$} \\
\cline { 2 - 5 } & Hardness & Modulus of elasticity & Hardness & Modulus of elasticity \\
\cline { 2 - 5 } & GPa* & GPa* $^{*}$ & GPa & GPa* \\
\hline 1 & 0.80 & 22.11 & 0.50 & 13.80 \\
\hline 3 & 0.79 & 21.19 & 0.64 & 18.29 \\
\hline 4 & 0.77 & 20.70 & 0.53 & 15.29 \\
\hline 5 & 0.68 & 23.36 & 0.52 & 14.69 \\
\hline Mean & 0.71 & 18.98 & 0.69 & 22.79 \\
\hline SD & 0.75 & 21.27 & 0.58 & 16.97 \\
\hline
\end{tabular}

${ }^{*} \mathrm{GPa}$ is Giga Pascal; $(\mathrm{P}<0.02)$ for Hardness; $\mathrm{P}=(\mathrm{NS})$ for Modulus of Elasticity

Table 3: Hardness values $\mathrm{GPa}^{*}$ and Modulus of elasticity $\mathrm{GPa}^{\star}$ of control and corresponding experimental dentine samples at six months after treatment with non-setting calcium hydroxide.

\begin{tabular}{l|c|c|c|c}
\multirow{2}{*}{ Slabs } & \multicolumn{2}{|c|}{ Control Group $\mathbf{n = 5}$} & \multicolumn{2}{c}{ Experimental group $\mathbf{n = 5}$} \\
\cline { 2 - 5 } & Hardness & Elastic module & Hardness & Elastic module \\
\cline { 2 - 5 } & GPa* & GPa $^{*}$ & GPa* $^{*}$ & 0.14 \\
\hline 1 & 0.69 & 22.80 & 0.20 & 05.65 \\
\hline 2 & 0.55 & 22.22 & 0.16 & 07.23 \\
\hline 4 & 0.79 & 21.19 & 0.25 & 07.59 \\
\hline 5 & 0.66 & 24.62 & 0.30 & 18.29 \\
\hline Mean & 0.76 & 20.24 & 0.21 & 09.09 \\
\hline SD & 0.69 & 22.21 & 0.065 & 05.19
\end{tabular}

*GPa is Giga Pascal; $(P<0.01)$ for Hardness; $(P<0.01)$ for Modulus of Elasticity 
was changed every week. All Petri dishes containing the samples were kept in an incubator at $37 \mathrm{C}^{\circ}$ to replicate oral temperature. Balooch et al. [1998] stated that non-hydrated dentine had a higher hardness and elasticity compared with hydrated dentine. Therefore, by creating a humid environment in this model, dentine hydration was ensured which would allow some extrapolation of these results to clinical situations [Angker and Swain, 2006]. The choice of the type of indenter for nanoindentation is also important. Angker and Swain [2006] have shown that a 5-20 $\mathrm{mm}$ spherical indenter is more reliable for the investigation of the enamel and dentine elasticity, as was used here.

It is clear from the results of nanoindentation that the hardness of dentine was significantly lower in the group where $\mathrm{NSCH}$ was applied as compared with the controls after 3 months. A reduction of roughly $50 \%$ was observed when the two means were compared. This difference was even more significant when the results were compared after 6 months when the hardness values for the experimental group were a third that of the control group. The modulus of elasticity of the experimental group was less than half that of the control samples. A decreased hardness and a reduced modulus of elasticity would signify a compromised dentine fracture resistance.

Although this was a pilot study with a relatively small sample size the statistically significant differences between the time points seem to be quite indicative of the detrimental effect of $\mathrm{NSCH}$ on the fracture resistance of dentine as a function of contact time. A recent study [Rosenberg et al., 2007] has also shown a significant decrease in the mean tensile fracture strength (MTFS) of dentine after the application of $\mathrm{Ca}(\mathrm{OH})_{2}$ for 84 days.

This study does not agree with a previous one by Yoldas et al. [2004], where a statistically significant decrease in hardness of dentine was shown as soon as 7 days after the application of $\mathrm{NSCH}$. From the present study design it can only be concluded that the significant changes started to manifest sometime between 1 and 3 months. It would have been interesting to carry out the hardness measurements at more regular intervals to pinpoint exactly the time at which the changes start to become clinically relevant.

It is possible that $\mathrm{NSCH}$ causes a change in matrix content, denatures proteins, affects the $\mathrm{pH}$ or changes the mechanical and chemical properties of root dentine walls [White et al., 2002; Andreasen et al., 2002; Doyon et al., 2005; Andreasen et al., 2006a; Rosenberg et al., 2007]. Andreasen et al. [2002] in their study on sheep teeth concluded that it was indeed the high $\mathrm{pH}$ of $\mathrm{NSCH}$ that caused proteolysis and weakened the dentine by up to $50 \%$ that might lead to root fracture. White et al. [2002] reported a 32\% decrease in dentine strength after use of $\mathrm{Ca}(\mathrm{OH})_{2}$. Yoldas et al. [2004] showed that the use of $\mathrm{Ca}(\mathrm{OH})_{2}$ combinations for intra-canal dressing softened the dentine.

Despite the weight of evidence supporting the softening of dentine with application of $\mathrm{NSCH}$, caution should be exer- cised when extrapolating between different experiments as there are clear variations in protocols, e.g. teeth stored at $22 C^{\circ}$ [Doyon et al., 2005], or the use in one study of sodium hypochlorite as an irrigation solution before the application of $\mathrm{Ca}(\mathrm{OH})_{2}$ [Rosenberg et al., 2007]. Sodium hypochlorite has been proven by many studies to have a negative affect on dentine hardness [Sim et al., 2001; White et al., 2002].

The effect of $\mathrm{NSCH}$ on the proteins in dentine was not determined in this study, but it can be assumed that a reduced hardness and modulus of elasticity could possibly be a result of denaturing of proteins within the dentine matrix. Certainly the SEM images showed widespread distribution of $\mathrm{NSCH}$ inside the dentinal tubules throughout the dentine after 3 and 6 months of application. It has also been shown that the hardness of dentine is also directly related to its mineral content [Marshall et al. 2001; Angkera et al. 2004]. Further study is required to understand the mechanism through which the contact of $\mathrm{NSCH}$ with dentine compromises its strength and fracture resistance.

The material used in this study was an aqueous NSCH (UltraCal) that is a powerful antibacterial agent, a property derived from its intensely alkaline nature. It has been reported that aqueous $\mathrm{NSCH}$ pastes such as Ultra-Cal $\AA$ have a higher dentinal $\mathrm{pH}$ than any other forms of $\mathrm{Ca}(\mathrm{OH})_{2}$ [Pérez et al., 2001, Zmener et al, 2007]. This might explain the proteolytic action of $\mathrm{Ca}(\mathrm{OH})_{2}$ as previously reported [Andreasen et al., 2002] and also explains the results of this present study.

Clinical implications. NSCH is often used for prolonged periods in root canals for disinfection and for the establishment of a hard tissue barrier to facilitate root canal obturation in non-vital immature incisors. However, this present study suggests that a long-term use of $\mathrm{NSCH}$ may have a detrimental effect on the hardness and modulus of elasticity of root dentine possibly making it more prone to root fracture through a decrease in fracture resistance. $\mathrm{NSCH}$ continues to be a popular material and widely used in endodontic treatment of non-vital young permanent incisors. However, clinicians should take note of these findings and those recently published and make endeavors to limit the period of time $\mathrm{NSCH}$ is placed in the root canals. If $\mathrm{NSCH}$ is used for disinfection of root canals then it is unlikely to be used for prolonged periods. The prolonged use is usually for inducing hard tissue barrier formation and that can take many months to accomplish [Kinirons et al., 2001]. The use of newer materials such as mineral trioxide aggregate (MTA) should be considered for the creation of the hard tissue barrier, as this would eliminate the use of $\mathrm{NSCH}$ for prolonged periods. However, MTA itself is a highly alkaline material, and before it can confidently be recommend that dentists use MTA as an alternative to $\mathrm{NSCH}$, more studies on its effect on the integrity and properties of dentine are required. 


\section{Conclusion}

There was a significant reduction in the hardness and Modulus of elasticity dentine with NSCH application. This reduction was clearly time dependent. There was no obvious disruption of dentine or tubular arrangement in SEM examination of dentine samples one, three or six months after application of $\mathrm{NSCH}$. Widespread distribution of $\mathrm{NSCH}$ was seen inside the dentinal tubules three and six months after the application of $\mathrm{NSCH}$. It is suggested that after six months of $\mathrm{NSCH}$ application, this softer, less stiff dentine may be less able to withstand masticatory forces, making the dentine more prone to fracture.

\section{Acknowledgements}

This paper was presented at the 9th Congress of the European Academy of Paediatric Dentistry in June 2008 and was a winner of a EAPD Young Scientist Award.

\section{References}

Al-Jundi SH. Type of treatment, prognosis and estimation of time spent to manage dental trauma in late presentation cases at a dental teaching hospital: a longitudinal and retrospective study. Dental Traumatol 2004;20:1-5.

Andreasen JO, Farik B, Munksgaard EC. Long-term calcium hydroxide as a root canal dressing may increase risk of root fracture. Dental Traumatol, 2002;18:134-7.

Andreasen JO, Bakland LK, Matras RC. Andreasen F M. traumatic intrusion of permanent teeth part 1 . An epidemiological study of 216 intruded permanent teeth. Dental Traumatol, 2006a;22:83-89.

Andreasen JO, Munksgaard EC, Bakland LK. Comparison of fracture resistance in root canals of immature sheep teeth after filling with calcium hydroxide or MTA. Dental Traumatol, 2006b;18:134-137.

Angkera L, Nockoldsb C, Swainac M, Kilpatrickd N.Correlating the mechanical properties to the mineral content of carious dentine-a comparative study using an ultra-micro indentation system (UMIS) and SEM-BSE signals. Anhs Oral Bial 2004; 49:369-378.

Angker L, Swain MV. Nanoindentation: Aplication to dental hard tissue investigations. J Material Research, 2006; 21:1893-1905.

Balooch M, Wu-Magidi IC, Balazs A, et al. Viscoelastic properties of demineralized human dentin measured in water with atomic force microscope (AFM)-based indentation. J Biomed Material Res, 1998;40:539-543.
Chng HK, Palamara JE, Messer, HH. Effect of hydrogen peroxide and sodium perborate on biomechanical properties of human dentine. J Endodont, 2002;28:62-67.

Cvek M. Prognosis of luxated non-vital maxillary incisors treated with calcium hydroxide and filled with gutta-percha. Endodont Dental Traumatol, 1992 8:45-55.

Doyon GE, Dumsha T, Von Fraunhofer JA. Fracture Resistance of Human Root Dentine Exposed to Intracanal Calcium Hydroxide. J Endodont, 2005;31:895-897.

Frank AL. Therapy for the divergent pulpless tooth by continued apical formation. J Amer Dent Assoc, 1966;72:87-93.

Habelitz S, Marshall GW, Balooch M and Marshal SJ. Nanoindentation and storage of teeth. J Biomaterial Res, 2000;35:995-998.

Hermann BW. (1920) Calcium Hydroxide as a medicament for the treatment and filling of root canals (German). Dissertation. University of Wurzburg, Germany. 1920.

Kaiser HJ. Mangement of wide open canals with calcium hydroxide. Twenty-first annual meeting of American Association of Endodontics, USA. Washington, DC, 1964.

Kinirons MJ, Srinivasan. V, Welbury RR, Finucane D. A study in two centres of variations in the time of apical barrier detection and barrier position in nonvital immature permanent incisors. Int J Paediatr Dent, 2001;11:447-451.

Marshall G, Habelitzl S, Gallagher R, et al. Nanomechanical Properties of Hydrated Carious Human Dentin. J Dent Res, 2001;80:1768-1771.

Oliver WC Pharr G.M. An improved technique for determining hardness and elastic modulus using load and displacement sensing indentation experiments. J Material Search, 1992;7:1564-1583.

Pérez F, Franchi M, Péli JF. Effect of calcium hydroxide form and placement on root dentine $\mathrm{pH}$. Int Endodo J, 2001;34:417-423.

Rosenberg B, Murray PE, Namerow K. The effect of calcium hydroxide root filling on dentin fracture strength. Dental Traumatol, 2007;23:26-29.

Sim TP Knowles Y, Shelton J, Gulabivala K. Effect of sodium hypochlorite on mechanical properties of dentine and tooth surface strain. J Material Res, 2001;34:120-132.

Toparli M Koksal N. Hardness and yield strength of dentin from simulated nano-indentation tests Computer Methods and Programs in Biomedicine. 2005;77:253-257

White JD, Lacefield WR, Chavers LS, Eleazer PD. The effect of three commonly used endodontic materials on the strength and hardness of root dentin. J Endodo, 2002;28:828-830.

Yoldas O, Dogan C, Seydaoglu G. The effect of two different calcium hydroxide combinations on root dentine microhardness. Int Endo J, 2004;37:828831.

Zmener O, Pameijer $\mathrm{CH}$, Banegas $\mathrm{G}$. An in vitro study of the $\mathrm{pH}$ of three calcium hydroxide dressing materials. Dental Traumatol 2007;23:21-25. 